\title{
Asymptotic normality of quadratic forms of martingale differences
}

\author{
Liudas Giraitis $^{1}$ - Masanobu Taniguchi ${ }^{2}$. \\ Murad S. Taqqu ${ }^{3}$
}

Received: 31 May 2016 / Accepted: 23 June 2016 / Published online: 18 July 2016

(C) The Author(s) 2016. This article is published with open access at Springerlink.com

\begin{abstract}
We establish the asymptotic normality of a quadratic form $Q_{n}$ in martingale difference random variables $\eta_{t}$ when the weight matrix $A$ of the quadratic form has an asymptotically vanishing diagonal. Such a result has numerous potential applications in time series analysis. While for i.i.d. random variables $\eta_{t}$, asymptotic normality holds under condition $\|A\|_{s p}=o(\|A\|)$, where $\|A\|_{s p}$ and $\|A\|$ are the spectral and Euclidean norms of the matrix $A$, respectively, finding corresponding sufficient conditions in the case of martingale differences $\eta_{t}$ has been an important open problem. We provide such sufficient conditions in this paper.
\end{abstract}

Keywords Asymptotic normality · Quadratic form · Martingale differences

Mathematics Subject classification 62E20 - 60F05

Liudas Giraitis

L.Giraitis@qmul.ac.uk

Masanobu Taniguchi

taniguchi@waseda.jp

Murad S. Taqqu

murad@bu.edu

1 School of Economics and Finance, Queen Mary University of London, Mile End Road, London W2 3PR, UK

2 Research Institute for Science and Engineering, Waseda University, 3-4-1 Okubo, Shinjuku-ku, Tokyo 169-8555, Japan

3 Department of Mathematics and Statistics, Boston University, 111 Cummington Mall, Boston, MA 02215, USA 


\section{Main results}

We study here quadratic forms

$$
Q_{n}=\sum_{t, k=1}^{n} a_{n ; t k} \eta_{t} \eta_{k}
$$

where $\left\{\eta_{k}\right\}$ is a stationary ergodic martingale difference (m.d.) sequence with respect to some natural filtration $\mathcal{F}_{t}$, with moments

$$
E \eta_{k}=0, \quad E \eta_{k}^{2}=1 \text { and } E \eta_{k}^{4}<\infty .
$$

The real-valued coefficients $a_{n ; t k}$ in (1.1) are entries of a symmetric matrix $A_{n}=$ $\left(a_{n ; t k}\right)_{t, k=1, \ldots, n}$. We denote by

$$
\left\|A_{n}\right\|=\left(\sum_{t, k=1}^{n} a_{n ; t k}^{2}\right)^{1 / 2}
$$

the Euclidean norm and by

$$
\left\|A_{n}\right\|_{s p}=\max _{\|x\|=1}\left\|A_{n} x\right\|
$$

the spectral norm of the matrix $A_{n}$. For convenience, we set $a_{n ; t k}=0$ for $t \leq 0, t>n$ or $k \leq 0, k>n$.

The asymptotic normality property of the quadratic form $Q_{n}$ has been well investigated when the random variables $\eta_{j}$ are i.i.d. If $A_{n}$ has vanishing diagonal: $a_{n ; t}=0$ for all $t$, then asymptotic normality is implied by the condition

$$
\left\|A_{n}\right\|_{s p}=o\left(\left\|A_{n}\right\|\right),
$$

see Rotar (1973), De Jong (1987), Guttorp and Lockhart (1988), Mikosch (1991) and Bhansali et al. (2007a).

The aim of this paper is to extend these results to the m.d. noise $\eta_{j}$. We will need the following additional assumptions on the m.d. noise $\eta_{t}$ :

$$
E\left(\eta_{j}^{2} \mid \mathcal{F}_{j-1}\right) \geq c>0, \quad(\exists c>0) .
$$

The assumption (1.3) bounds the conditional variance of $\eta_{j}$ away from zero. We also assume that $A_{n}$ has an asymptotically "vanishing" diagonal in the sense:

$$
\sum_{t=1}^{n}\left|a_{n ; t t}\right|=o\left(\left\|A_{n}\right\|\right), \quad n \rightarrow \infty .
$$

Relation (1.4) implies

$$
\sum_{t=1}^{n} a_{n ; t t}^{2}=o\left(\left\|A_{n}\right\|^{2}\right), \quad n \rightarrow \infty .
$$

The following theorem shows that in case of m.d. noise $\left\{\eta_{k}\right\}$, the condition

$$
\left\|A_{n}\right\|_{s p} /\left\|A_{n}\right\| \rightarrow 0
$$

above needs to be strengthened by including the assumptions (1.8) and (1.9) on the weights $a_{n ; t s}$. Its proof is based on the martingale central limit theorem. 
Theorem 1.1 Let $Q_{n}$ be as in (1.1), where $\left\{\eta_{j}\right\}$ is a stationary ergodic m.d. noise such that $E \eta_{j}^{4}<\infty$ and (1.3) hold. Suppose that the $a_{n ; t s}$ 's are such that, as $n \rightarrow \infty$,

$$
\left\|A_{n}\right\|_{s p} /\left\|A_{n}\right\| \rightarrow 0 .
$$

Then there exist $c_{1}, c_{2}>0$ such that

$$
c_{1}\left\|A_{n}\right\|^{2} \leq \operatorname{Var}\left(Q_{n}\right) \leq c_{2}\left\|A_{n}\right\|^{2}, \quad n \geq 1 .
$$

If in addition,

$$
\sum_{t, s=1:|t-s| \geq L}^{n} a_{n ; t s}^{2}=o\left(\left\|A_{n}\right\|^{2}\right), \quad n \rightarrow \infty, \quad L \rightarrow \infty,
$$

and

$$
\sum_{t=k+2}^{n}\left(a_{n ; t, t-k}-a_{n ; t-1, t-1-k}\right)^{2}=o\left(\left\|A_{n}\right\|^{2}\right), \quad \forall k \geq 1
$$

then the following normal convergence holds:

$$
\left(\operatorname{Var}\left(Q_{n}\right)\right)^{-1 / 2}\left(Q_{n}-E Q_{n}\right) \stackrel{d}{\rightarrow} N(0,1)
$$

As usual, “ $\stackrel{d}{\rightarrow} N(0,1)$ " denotes convergence in distribution to a normal random variable with mean zero and variance one.

Theorem 1.1 plays an important instrumental role in establishing asymptotic properties of various estimation and testing procedures in parametric and non-parametric time series analysis where the object of interest can be written as a quadratic form

$$
Q_{n, X}=\sum_{t, s=1}^{n} e_{n}(t-s) X_{t} X_{s}
$$

of a linear (moving-average) process

$$
X_{t}=\sum_{j=0}^{\infty} a_{j} \eta_{t-j}
$$

of uncorrelated noise $\eta_{t}$ and the weights $e_{n}(s)$ may depend on $n$. In the case of i.i.d. noise $\eta_{t}$, the asymptotic normality for $Q_{n, X}$ is established by approximating it by a simpler quadratic form

$$
Q_{n, \eta}=\sum_{t, s=1}^{n} b_{n}(t-s) \eta_{t} \eta_{s}
$$

with some different weights $b_{n}(t)$ and then deriving the asymptotic normality for $Q_{n, \eta}$, as in Bhansali et al. (2007b). For example, one sets

$$
b_{n}(t)=\int_{-\pi}^{\pi} u_{n}(x) f(x) e^{i t x} d x
$$

where $f(x)$ is the spectral density of the sequence $X_{t}$, and where $u_{n}(x)$ is some convenient function related to $e_{n}(t)$, typically such that

$$
e_{n}(t)=\int_{-\pi}^{\pi} u_{n}(x) e^{i t x} d x .
$$


In general, obtaining simple asymptotic normality conditions for $Q_{n, X}$ is a hard theoretical problem but of great practical importance, which for an i.i.d. noise $\eta_{t}$ was solved in Bhansali et al. (2007b). In addition, in Sect. 6.2 in Giraitis et al. (2012) one considers discreet frequencies and shows that a sum

$$
S_{n}=\sum_{j=1}^{n / 2} b_{n j} I\left(u_{j}\right)
$$

of weighted periodograms

$$
I\left(u_{j}\right)=(2 \pi n)^{-1}\left|\sum_{k=1}^{n} e^{i k u_{j}} X_{k}\right|^{2}
$$

of the sequence $X_{t}$ at Fourier frequencies $u_{j}$ can be also effectively approximated by a quadratic form $Q_{n, \eta}$. This allows, by theorem like Theorem 1.1, to establish the asymptotic normality for such sums $S_{n}$. However, assumption of i.i.d. noise is restrictive and may be not satisfied in practical applications and in some theoretical, i.e. ARCH, settings. In a subsequent paper we will derive corresponding normal approximation results for $Q_{n, X}$ and $S_{n}$ when $\eta_{t}$ is a martingale difference process.

The following Corollary 1.1 displays situations where the conditions of Theorem 1.1 are easily satisfied. For a Toeplitz matrix $A_{n}$, that is with entries

$$
a_{n ; t s}=b_{n}(t-s),
$$

the assumption (1.9) is clearly satisfied, since

$$
a_{n ; t, t-k}-a_{n ; t-1, t-1-k}=b_{n}(k)-b_{n}(k)=0 .
$$

The following lemma provides a useful bound that can be used to prove (1.6).

Lemma 1.1 Suppose that

$$
b_{n}(t)=\int_{-\pi}^{\pi} e^{i t x} g_{n}(x) d x, \quad t=0,1, \ldots,
$$

where $g_{n}(x),|x| \leq \pi$ is an even real function. If there exists

$$
0 \leq \alpha<1 / 2
$$

and a sequence of constants $k_{n}>0$ such that

$$
\left|g_{n}(x)\right| \leq k_{n}|x|^{-\alpha}, \quad|x| \leq \pi,
$$

then

$$
\left\|A_{n}\right\|_{s p} \leq C k_{n} n^{\alpha}, \quad n \geq 1 .
$$

For the proof see Theorem 2.2(i) in Bhansali et al. (2007a).

Suppose now, in addition, that $g_{n}(x) \equiv g(x), n \geq 1$ and $|g(x)| \leq C|x|^{-\alpha},|x| \leq \pi$. Then

$$
\int_{-\pi}^{\pi} g^{2}(x) d x<\infty, \quad b_{n}(t)=b(t) \text { and } \sum_{t=-\infty}^{\infty} b^{2}(t)<\infty
$$

and, in addition, $k_{n}=1$ in (1.11). Hence

$$
\|A\|^{2}=\sum_{t, s=1}^{n} b^{2}(t-s)=\sum_{k=-n}^{n} b^{2}(k)(n-|k|) \sim n \sum_{t=-\infty}^{\infty} b^{2}(t) \text { as } n \rightarrow \infty
$$


and

$$
\left\|A_{n}\right\|_{s p} \leq C n^{\alpha}=o\left(n^{1 / 2}\right)=o(\|A\|)
$$

which implies (1.6). Moreover,

$$
\sum_{t, s=1:|t-s| \geq L}^{n} a_{n ; t s}^{2}=\sum_{t, s=1:|t-s| \geq L}^{n} b^{2}(t-s) \leq n \sum_{|k| \geq L} b^{2}(|k|) .
$$

Since $\sum_{|k| \geq L} b^{2}(|k|) \rightarrow 0$ as $L \rightarrow \infty$, we obtain (1.8). This together with Theorem 1.1 implies the following corollary.

\section{Corollary 1.1 Let}

$$
Q_{n}=\sum_{t, k=1}^{n} b(t-k) \eta_{t} \eta_{k}
$$

where $b(t)=b(-t), b(0)=0$ are real weights and $\left\{\eta_{j}\right\}$ is a stationary ergodic m.d. noise such that $E \eta_{j}^{4}<\infty$ and (1.3) hold.

(i) If $\sum_{t=0}^{\infty}|b(t)|<\infty$, then

$$
\begin{aligned}
& \exists c_{1}, c_{2}>0: c_{1} n \leq \operatorname{Var}\left(Q_{n}\right) \leq c_{2} n, \quad n \geq 1, \\
& \left(\operatorname{Var}\left(Q_{n}\right)\right)^{-1 / 2}\left(Q_{n}-E Q_{n}\right) \stackrel{d}{\rightarrow} N(0,1) .
\end{aligned}
$$

(ii) If $b(t)=\int_{-\pi}^{\pi} e^{i t x} g(x) d x, t=0,1, \ldots$, where $g(x),|x| \leq \pi$ is an even real function such that for some $0 \leq \alpha<1 / 2$ and $C>0$,

$$
|g(x)| \leq C|x|^{-\alpha}, \quad|x| \leq \pi
$$

then (1.12) and (1.13) hold.

Next we consider two quadratic forms

$$
\begin{aligned}
& Q_{n}^{(1)}=\sum_{t, s=1}^{n} a_{n ; t s}^{(1)} \eta_{t} \eta_{s}, \quad \text { and } \\
& Q_{n}^{(2)}=\sum_{t, s=1}^{n} a_{n ; t s}^{(2)} \eta_{t} \eta_{s},
\end{aligned}
$$

with corresponding matrices $A_{n}^{(1)}, A_{n}^{(2)}$ and a m.d. sequence $\eta_{t}$ which satisfy the assumptions of Theorem 1.1, so that

$$
\left(\operatorname{Var}\left(Q_{n}^{(i)}\right)\right)^{-1 / 2}\left(Q_{n}^{(i)}-E Q_{n}^{(i)}\right) \stackrel{d}{\rightarrow} N(0,1), \quad i=1,2 .
$$

The next corollary provides additional sufficient condition that implies asymptotic normality of their sum.

Corollary 1.2 Suppose that the quadratic forms $Q_{n}^{(1)}, Q_{n}^{(2)}$ in (1.15) satisfy the assumptions of Theorem 1.1. Set

$$
A_{n}=A_{n}^{(1)}+A_{n}^{(2)}
$$


If in addition

$$
\lim _{n \rightarrow \infty}\left\|A_{n}^{(1)}\right\|^{-1}\left\|A_{n}^{(2)}\right\|^{-1} \operatorname{tr}\left(A_{n}^{(1)} A_{n}^{(2)}\right)=0
$$

then the quadratic form $Q_{n}:=Q_{n}^{(1)}+Q_{n}^{(2)}$ satisfies

$$
\exists c_{1}, c_{2}>0: \quad c_{1}\left(\left\|A_{n}^{(1)}\right\|+\left\|A_{n}^{(2)}\right\|\right) \leq \operatorname{Var}\left(Q_{n}\right) \leq c_{2}\left(\left\|A_{n}^{(1)}\right\|+\left\|A_{n}^{(2)}\right\|\right), \quad n \geq 1,
$$

and

$$
\left(\operatorname{Var}\left(Q_{n}\right)\right)^{-1 / 2}\left(Q_{n}-E Q_{n}\right) \stackrel{d}{\rightarrow} N(0,1)
$$

Proof We have $Q_{n}=\sum_{t, s=1}^{n} a_{n ; t s} \eta_{t} \eta_{s}$ where $a_{n ; t s}=a_{n ; t s}^{(1)}+a_{n ; t s}^{(2)}$. Thus, to prove the corollary, it suffices to show that $A_{n}$ satisfies assumptions of Theorem 1.1. This easily follows from the fact that both $A_{n}^{(1)}$ and $A_{n}^{(2)}$ satisfy assumptions of Theorem 1.1, and the property

$$
\left\|A_{n}\right\|^{2}=\left(\left\|A_{n}^{(1)}\right\|^{2}+\left\|A_{n}^{(2)}\right\|^{2}\right)(1+o(1)) .
$$

The latter follows from

$$
\left\|A_{n}\right\|^{2}=\left\|A_{n}^{(1)}\right\|^{2}+\left\|A_{n}^{(2)}\right\|^{2}+2 \operatorname{tr}\left(A_{n}^{(1)} A_{n}^{(2)}\right)
$$

because the matrices $A_{n}^{(1)}$ and $A_{n}^{(2)}$ are symmetric so the cross term

$$
2 \sum_{t, s} a_{n ; t s}^{(1)} a_{n ; t s}^{(2)}=2 \sum_{t, s} a_{n ; t s}^{(1)} a_{n ; s t}^{(2)}=2 \operatorname{tr}\left(A_{n}^{(1)} A_{n}^{(2)}\right) .
$$

Hence

$$
\left\|A_{n}\right\|^{2}=\left(\left\|A_{n}^{(1)}\right\|^{2}+\left\|A_{n}^{(2)}\right\|^{2}\right)\left(1+r_{n}\right)
$$

where

$$
r_{n}=2 \operatorname{tr}\left(A_{n}^{(1)} A_{n}^{(2)}\right) /\left(\left\|A_{n}^{(1)}\right\|^{2}+\left\|A_{n}^{(2)}\right\|^{2}\right) .
$$

Since $\left\|A_{n}^{(1)}\right\|^{2}+\left\|A_{n}^{(2)}\right\|^{2} \geq 2\left\|A_{n}^{(1)}\right\|\left\|A_{n}^{(2)}\right\|$ we get $r_{n}=o(1)$ by (1.16).

Corollary 1.2 indicates that we need the additional condition (1.16) in order to obtain the asymptotic normality of $Q_{n}$. It does not imply that in this case the components $Q_{n}^{(1)}$ and $Q_{n}^{(2)}$ are asymptotically uncorrelated and hence asymptotically independent. We conjecture that $Q_{n}^{(1)}$ and $Q_{n}^{(2)}$ will be asymptotically independent in the case when $\eta_{t}$ is an i.i.d. noise.

\section{Proof of Theorem 1.1}

In the proof of Theorem 1.1 we shall use the following result.

Lemma 2.1 (Dalla et al. (2014), Lemma 10). 
(i) Let

$$
T_{n}=\sum_{j \in Z} c_{n j} V_{j},
$$

where $\left\{V_{j}\right\}, j \in Z=\{\cdots,-1,0,1, \cdots\}$ is a stationary ergodic sequence, $E\left|V_{1}\right|<\infty$, and $c_{n j}$ are real numbers such that for some $0<\alpha_{n}<\infty, n \geq 1$,

$$
\sum_{j \in Z}\left|c_{n j}\right|=O\left(\alpha_{n}\right), \quad \sum_{j \in Z}\left|c_{n j}-c_{n, j-1}\right|=o\left(\alpha_{n}\right) .
$$

Then

$$
E\left|T_{n}-E T_{n}\right|=o\left(\alpha_{n}\right) .
$$

In particular, if $\alpha_{n}=1$, then

$$
T_{n}=E T_{n}+o_{p}(1) .
$$

(ii) If the m.d. sequence $\eta_{t}$ satisfies $\max _{t} E\left|\eta_{t}\right|^{p}<\infty$, for some $p \geq 2$, then

$$
E\left|\sum_{j \in Z} d_{j} \eta_{j}\right|^{p} \leq C\left(\sum_{j \in Z} d_{j}^{2}\right)^{p / 2}
$$

for any $d_{j}$ 's such that $\sum_{j \in Z} d_{j}^{2}<\infty$, where $C<\infty$ does not depend on $d_{j}$ 's.

For the convenience of the reader we provide the proof of the following lemma.

Lemma 2.2 One has

$$
\max _{t=1, \ldots, n} \sum_{s=1}^{n} a_{n ; t s}^{2} \leq\left\|A_{n}\right\|_{s p}^{2}, \quad \max _{t, s=1, \ldots, n}\left|a_{n ; t s}\right| \leq\left\|A_{n}\right\|_{s p} .
$$

Proof We drop the index $n$ and let $A=\left(a_{t s}\right)$. The second inequality $\left|a_{t s}\right| \leq \| A_{n}||_{s p}$ follows from the first since

$$
\max _{t, s} a_{t s}^{2} \leq \max _{t} \sum_{s=1}^{n} a_{t s}^{2} \leq\left\|A_{n}\right\|_{s p}^{2}
$$

Turning to the first inequality, we have $\left\|A_{n}\right\|_{s p}^{2}=\sup _{\|x\|=1}\|A x\|^{2}$ where $x=\left(x_{1}, \ldots, x_{n}\right)^{\prime}$ and

$$
\|A x\|^{2}=\left\|\sum_{s=1}^{n} a_{1 s} x_{s}, \ldots, \sum_{s=1}^{n} a_{n s} x_{s}\right\|^{2}=\left(\sum_{s=1}^{n} a_{1 s} x_{s}\right)^{2}+\cdots+\left(\sum_{s=1}^{n} a_{n s} x_{s}\right)^{2} .
$$

Set $y=(0, \ldots, 0,1,0, \ldots, 0)^{\prime}$ where 1 is at the $t_{0}$ position. Note that $\|y\|=1$. Then

$$
\left\|A_{n}\right\|_{s p}^{2} \geq\|A y\|^{2}=a_{1 t_{0}}^{2}+\cdots+a_{n t_{0}}^{2}=\sum_{s=1}^{n} a_{s t_{0}}^{2}=\sum_{s=1}^{n} a_{t_{0} s}^{2}
$$

since $A$ is symmetric. Hence

$$
\left\|A_{n}\right\|_{s p}^{2} \geq \max _{t_{0}=1, \ldots, n} \sum_{s=1}^{n} a_{t_{0} s}^{2}
$$


Proof of Theorem 1.1 Using (1.6), the second claim of (2.3) implies

$$
\max _{1 \leq k, u \leq L}\left|a_{n ; k u}\right|=o(\|A\|), \quad \forall L \geq 1 \text { fixed. }
$$

Relation (2.4) implies that no single $a_{n ; k u}$ dominates.

- Proof of (1.7) Below we write $a_{t s}$ instead of $a_{n ; t s}$. Let

$$
z_{n t}=2 \eta_{t} \sum_{s=1}^{t-1} a_{t s} \eta_{s} \quad \text { and } \quad z_{t}^{\prime}=a_{t t}\left(\eta_{t}^{2}-E \eta_{t}^{2}\right)
$$

Then

$$
Q_{n}-E Q_{n}=\sum_{t=2}^{n} z_{n t}+\sum_{t=1}^{n} z_{n t}^{\prime}=S_{n}+S_{n}^{\prime} .
$$

Observe that $E \eta_{t} \eta_{s}=0$ for $t>s$ and hence $E S_{n}=0$ since $\eta_{s}$ is a m.d. sequence. In addition,

$$
E S_{n}^{2}=4 \sum_{t=2}^{n} E\left[\eta_{t}^{2}\left(\sum_{s=1}^{t-1} a_{t s} \eta_{s}\right)^{2}\right]
$$

Using $E \eta_{t}^{4} \leq C$ and (1.4),

$$
E\left|S_{n}^{\prime}\right| \leq C \sum_{t=1}^{n}\left|a_{t t}\right|=o\left(\left\|A_{n}\right\|\right), \quad E S_{n}^{\prime 2} \leq C\left(\sum_{t=1}^{n}\left|a_{t t}\right|\right)^{2}=o\left(\left\|A_{n}\right\|^{2}\right) .
$$

Now we show that

$$
c_{1}\left\|A_{n}\right\|^{2} \leq E S_{n}^{2} \leq c_{2}\left\|A_{n}\right\|^{2} .
$$

The lower bound follows by using (1.3) and (1.5) because of the fact that $c>0$ :

$$
\begin{aligned}
E S_{n}^{2} & =4 \sum_{t=2}^{n} E\left[\eta_{t}^{2}\left(\sum_{s=1}^{t-1} a_{t s} \eta_{s}\right)^{2}\right]=4 \sum_{t=2}^{n} E\left[E\left[\eta_{t}^{2} \mid \mathcal{F}_{t-1}\right]\left(\sum_{s=1}^{t-1} a_{t s} \eta_{s}\right)^{2}\right] \\
& \geq 4 c \sum_{t=2}^{n} E\left(\sum_{s=1}^{t-1} a_{t s} \eta_{s}\right)^{2}=4 c \sum_{t=2}^{n} \sum_{s=1}^{t-1} a_{t s}^{2} \\
& =2 c \sum_{t, s=1}^{n} a_{t s}^{2}-2 c \sum_{t=1}^{n} a_{t t}^{2}=2\|A\|^{2}-o\left(\|A\|^{2}\right) \geq\|A\|^{2}
\end{aligned}
$$

for large $n$.

To prove the upper bound, notice that

$$
\begin{aligned}
E S_{n}^{2} & =4 \sum_{t=2}^{n} E\left[\eta_{t}^{2}\left(\sum_{s=1}^{t-1} a_{t s} \eta_{s}\right)^{2}\right] \\
& \left.\leq 4 \sum_{t=2}^{n}\left(E \eta_{t}^{4}\right)^{1 / 2}\left(E\left(\sum_{s=1}^{t-1} a_{t s} \eta_{s}\right)^{4}\right)^{1 / 2}\right] \leq C \sum_{t, s=1}^{n} a_{t s}^{2}=C\|A\|^{2}
\end{aligned}
$$


by (2.2) and assumption $E \eta_{t}^{4}=E \eta_{1}^{4}<\infty$. To obtain (1.7), note that

$$
\operatorname{Var}\left(Q_{n}\right) \leq 2 E S_{n}^{2}+2 E{S_{n}^{\prime}}^{2} \leq C\|A\|^{2}+o\left(\|A\|^{2}\right) \leq 2 C\|A\|^{2}
$$

by (2.8) and (2.10). In addition, (2.6)-(2.10) imply

$$
\operatorname{Var}\left(Q_{n}\right)=\left(E S_{n}^{2}\right)(1+o(1)), \quad n \rightarrow \infty .
$$

Indeed, by (2.6),

$$
\begin{aligned}
\left|\operatorname{Var}\left(Q_{n}\right)-\operatorname{Var}\left(S_{n}\right)\right| & =\left|\operatorname{Var}\left(S_{n}^{\prime}\right)+2 \operatorname{Cov}\left(S_{n}, S_{n}^{\prime}\right)\right| \leq \operatorname{Var}\left(S_{n}^{\prime}\right)+2\left(\operatorname{Var}\left(S_{n}\right) \operatorname{Var}\left(S_{n}^{\prime}\right)\right)^{1 / 2} \\
& =o\left(\|A\|^{2}\right)+\left(O\left(\|A\|^{2}\right) o\left(\|A\|^{2}\right)\right)^{1 / 2}=o\left(\|A\|^{2}\right)
\end{aligned}
$$

so that $\operatorname{Var}\left(Q_{n}\right)=\operatorname{Var}\left(S_{n}\right)+o\left(\|A\|^{2}\right)$ and by (2.9) we have $E S_{n}^{2} \geq\|A\|^{2}$, which leads to (2.11).

- Proof of (1.10) We now prove the asymptotic normality of $Q_{n}$. Let $B_{n}^{2}=\operatorname{Var}\left(Q_{n}\right)$, $X_{n t}=B_{n}^{-1} z_{n t}$ and $X_{t}^{\prime}=B_{n}^{-1} z_{n t}^{\prime}$. Then, by (2.6)

$$
B_{n}^{-1}\left(Q_{n}-E Q_{n}\right)=\sum_{t=2}^{n} X_{n t}+\sum_{t=1}^{n} X_{n t}^{\prime}
$$

Observe that by (1.7) and (2.8), $E\left|\sum_{t=1}^{n} X_{t}^{\prime}\right|=B_{n}^{-1} E\left|\sum_{s=1}^{n} z_{n t}^{\prime}\right| \leq C|| A_{n} \|^{-1} \sum_{t=1}^{n}\left|a_{t t}\right|=$ $o(1)$. Therefore, to prove (1.10) it remains to show that

$$
\sum_{t=2}^{n} X_{n t} \stackrel{d}{\rightarrow} N(0,1)
$$

Since $X_{n t}$ is a m.d. sequence, then by Theorem 3.2 of Hall and Heyde (1980), it suffices to show

$$
\text { (a) } E \max _{1 \leq j \leq n} X_{n j}^{2} \rightarrow 0, \quad \text { (b) } \max _{1 \leq j \leq n}\left|X_{n j}\right| \rightarrow_{p} 0, \quad \text { (c) } \sum_{j=1}^{n} X_{n j}^{2} \rightarrow_{p} 1 \text {. }
$$

•• To verify (a) and (b), it suffices to show that for any $\varepsilon>0$,

$$
\sum_{j=1}^{n} E X_{n j}^{2} I\left(\left|X_{n j}\right| \geq \varepsilon\right) \rightarrow 0
$$

which clearly implies (a), while (b) follows from (2.15) noting that

$$
P\left(\max _{1 \leq j \leq n}\left|X_{n j}\right| \geq \varepsilon\right) \leq \varepsilon^{-2} \sum_{j=1}^{n} E X_{n j}^{2} I\left(\left|X_{n j}\right| \geq \varepsilon\right) \rightarrow 0 .
$$

To prove (2.15), let $K>0$ be large. We consider two cases: $\eta_{t}^{2} \leq K$ and $\eta_{t}^{2}>K$. Then,

$$
\begin{aligned}
E X_{n t}^{2} I\left(X_{n t}^{2} \geq \varepsilon\right) I\left(\eta_{t}^{2} \leq K\right) & \leq \varepsilon^{-1} E X_{n j}^{4} I\left(\eta_{t}^{2} \leq K\right) \leq \varepsilon^{-1} 2^{4} K^{2} B_{n}^{-4} E\left(\sum_{s=1}^{t-1} a_{t s} \eta_{s}\right)^{4} \\
& \leq C \varepsilon^{-1} K^{2} B_{n}^{-4}\left(\sum_{s=1}^{t-1} a_{t s}^{2}\right)^{2} \leq C \varepsilon^{-1} K^{2} B_{n}^{-4}\|A\|_{s p}^{2} \sum_{s=1}^{t-1} a_{t s}^{2}
\end{aligned}
$$


by (2.2) and (2.3). Recall that by (1.7), $B_{n}^{-2} \leq C\|A\|^{-2}$. Thus, for any $\varepsilon>0$ and $K>0$,

$$
\begin{aligned}
\sum_{t=2}^{n} E X_{n t}^{2} I\left(X_{n t}^{2} \geq \varepsilon\right) I\left(\eta_{t}^{2} \leq K\right) & \leq C \varepsilon^{-1} K^{2} B_{n}^{-4}\|A\|_{s p}^{2} \sum_{t=2}^{n} \sum_{s=1}^{t-1} a_{t s}^{2} \\
& \leq C \varepsilon^{-1} K^{2}\left(\|A\|_{s p} /\|A\|\right)^{2} \rightarrow 0
\end{aligned}
$$

by (1.6) as $n \rightarrow \infty$ for any finite $K$.

We now focus on the case $\eta_{t}^{2} \geq K$. Since $E \eta_{t}^{4}<\infty$ and, by stationarity of $\eta_{t}, \delta_{K}:=$ $E \eta_{1}^{4} I\left(\eta_{1}^{2}>K\right) \rightarrow 0$ as $K \rightarrow \infty$, this implies

$$
\begin{aligned}
E X_{n t}^{2} I\left(X_{n t}^{2} \geq \varepsilon\right) I\left(\eta_{t}^{2}>K\right) & \leq E X_{n t}^{2} I\left(\eta_{t}^{2}>K\right) \leq B_{n}^{-2} 2^{2} E\left[\eta_{t}^{2} I\left(\eta_{t}^{2}>K\right)\left(\sum_{s=1}^{t-1} a_{t s} \eta_{s}\right)^{2}\right] \\
& \leq C\|A\|^{-2} \delta_{K}^{1 / 2}\left(E\left(\sum_{s=1}^{t-1} a_{t s} \eta_{s}\right)^{4}\right)^{1 / 2} \leq C\|A\|^{-2} \delta_{K}^{1 / 2} \sum_{s=1}^{t-1} a_{t s}^{2}
\end{aligned}
$$

by (2.2). Hence,

$$
\begin{aligned}
& \sum_{t=2}^{n} E X_{n t}^{2} I\left(X_{n t}^{2} \geq \varepsilon\right) I\left(\eta_{t}^{2}>K\right) \leq C \delta_{K}^{1 / 2}\|A\|^{-2} \sum_{t=2}^{n} \sum_{s=1}^{t-1} a_{t s}^{2} \\
& \leq C \delta_{K}^{1 / 2} \rightarrow 0, \quad K \rightarrow \infty .
\end{aligned}
$$

Since (2.16) holds for any fixed $K$ as $n \rightarrow \infty$, and since (2.17) holds as $K \rightarrow \infty$ uniformly in $n$, we get (2.15).

- The verification of (c) in (2.14) is particularly delicate. We want to show that $s_{n} \rightarrow{ }_{p} 1$. Recall that $x_{n t}=B^{-1} z_{n t}$ where $z_{n t}$ is defined in (2.5). We shall decompose $s_{n}=\sum_{s=1}^{n} X_{n s}^{2}$ into two parts involving $L>1$. Write

$$
s_{n}=4 B_{n}^{-2} \sum_{t=1}^{n} \eta_{t}^{2}\left(\sum_{s=1}^{t-1} a_{t s} \eta_{s}\right)^{2}=s_{n, 1}+s_{n, 2},
$$

where

$$
s_{n, 1}:=4 B_{n}^{-2} \sum_{t=1}^{n} \eta_{t}^{2}\left(\sum_{s=t-L}^{t-1} a_{t s} \eta_{s}\right)^{2}, \quad s_{n, 2}:=s_{n}-s_{n, 1}
$$

Then,

$$
s_{n}=E s_{n}+\left(s_{n, 1}-E s_{n, 1}\right)+\left(s_{n, 2}-E s_{n, 2}\right) .
$$

We show that as $n \rightarrow \infty$,

$$
\begin{aligned}
& \text { (i) } E s_{n} \rightarrow 1 ; \quad \text { (ii) } s_{n, 1}-E s_{n, 1} \rightarrow{ }_{p} 0, \quad \forall L \geq 1 ; \\
& \text { (iii) } E\left|s_{n, 2}\right| \rightarrow 0, \quad n \rightarrow \infty, \quad L \rightarrow \infty
\end{aligned}
$$

which proves (2.14)(c) since $E\left|s_{n}\right| \rightarrow 0$ implies $s_{n} \rightarrow{ }_{P} 0$ as $n \rightarrow \infty$ and $L \rightarrow \infty$.

• - The claim (2.19)(i) follows from (2.11),

$$
\left(E S_{n}^{2}\right) / \operatorname{Var}\left(Q_{n}\right)=B_{n}^{-2} E S_{n}^{2} \rightarrow 1,
$$


noting that $B_{n}^{-2} E S_{n}^{2}=E s_{n}$, which holds by definition of $s_{n}$ and (2.7).

$\bullet \bullet$ To show (2.19)(ii), open up the squares, set $s=t-k$ and $s^{\prime}=t-u$, to get

$s_{n, 1}-E s_{n, 1}=4 \sum_{k, u=1}^{L}\left\{B_{n}^{-2} \sum_{t=1}^{n} a_{t, t-k} a_{t, t-u}\left[\eta_{t}^{2} \eta_{t-k} \eta_{t-u}-E \eta_{t}^{2} \eta_{t-k} \eta_{t-u}\right]\right\}=4 \sum_{k, u=1}^{L} g_{n, k u}$.

It suffices to verify that for any fixed $k$ and $u, g_{n, k u}=o_{p}(1)$. Setting

$$
c_{n t}:=B_{n}^{-2} a_{t, t-k} a_{t, t-u}
$$

and

$$
V_{t}:=\eta_{t}^{2} \eta_{t-k} \eta_{t-u}-E \eta_{t}^{2} \eta_{t-k} \eta_{t-u}
$$

write

$$
g_{n, k u}=\sum_{t=1}^{n} c_{n t} V_{t}
$$

Since the noise $\left\{\eta_{t}\right\}$ is stationary ergodic and such that $E \eta_{1}^{4}<\infty$, by Theorem 3.5.8 in Stout (1974), the process $\left\{V_{j}\right\}$ is stationary and ergodic, and $E\left|V_{1}\right|<\infty$. Because of the centering, $E g_{n, k u}=0$. Thus, by Lemma 2.1(i), to prove $g_{n, k u}=o_{p}(1)$, it remains to show that $c_{n t}$ 's satisfy (2.1) with $\alpha_{n}=1$. Observe that

$$
\sum_{t \in Z}\left|c_{n t}\right|=B_{n}^{-2} \sum_{t=1}^{n}\left|a_{t, t-k} a_{t, t-u}\right| \leq 2 B_{n}^{-2} \sum_{t, s=1}^{n} a_{t, s}^{2}=2 B_{n}^{-2}\|A\|^{2} \leq C, \quad n \rightarrow \infty
$$

by (1.7). On the other hand,

$$
\begin{aligned}
& \sum_{t \in Z}\left|c_{n t}-c_{n, t-1}\right|=B_{n}^{-2} \sum_{t=1}^{n+1}\left|a_{t, t-k} a_{t, t-u}-a_{t-1, t-1-k} a_{t-1, t-1-u}\right| \\
& \leq B_{n}^{-2} \sum_{t=1}^{n+1}\left\{\left|a_{t, t-k}-a_{t-1, t-1-k}\right|\left|a_{t, t-u}\right|+\left|a_{t-1, t-1-k}\right|\left|a_{t, t-u}-a_{t-1, t-1-u}\right|\right\} \\
& \leq B_{n}^{-2}\left\{\left(\sum_{t=1}^{n+1}\left(a_{t, t-k}-a_{t-1, t-1-k}\right)^{2}\right)^{1 / 2}+\left(\sum_{t=1}^{n+1}\left(a_{t, t-u}-a_{t-1, t-1-u}\right)^{2}\right)^{1 / 2}\right\} \\
& \quad \times\left(\sum_{t, s=1}^{n} a_{t, s}^{2}\right)^{1 / 2} \\
& =o\left(B_{n}^{-2}\|A\|^{2}\right)=o(1),
\end{aligned}
$$

by (1.9), (2.3) and (1.7). Hence (2.1) holds. By Lemma 2.1(i) we conclude that $g_{n, k u}=o_{p}(1)$ and, thus, $s_{n, 1}-E s_{n, 1}=o_{p}(1)$. Hence (2.19)(ii) holds.

$\bullet \bullet \bullet$ To verify $E\left|s_{n, 2}\right| \rightarrow 0$ in (2.19)(iii), write

$$
s_{n, 2}=s_{n}-s_{n, 1}=4 B_{n}^{-2} \sum_{t=1}^{n} \eta_{t}^{2}\left[\left(\sum_{s=1}^{t-1} a_{t s} \eta_{s}\right)^{2}-\left(\sum_{s=t-L}^{t-1} a_{t s} \eta_{s}\right)^{2}\right] .
$$


We use the identity $a^{2}-b^{2}=(a-b)^{2}+2(a-b) b$, to obtain

$$
\begin{aligned}
\left|s_{n, 2}\right|= & 4 B_{n}^{-2}\left|\sum_{t=1}^{n} \eta_{t}^{2}\left\{\left(\sum_{s=1}^{t-1} a_{t s} \eta_{s}\right)^{2}-\left(\sum_{s=t-L}^{t-1} a_{t s} \eta_{s}\right)^{2}\right\}\right| \\
= & 4 B_{n}^{-2}\left|\sum_{t=1}^{n} \eta_{t}^{2}\left\{\left(\sum_{s=1}^{t-L-1} a_{t s} \eta_{s}\right)^{2}+2\left(\sum_{s=1}^{t-L-1} a_{t s} \eta_{s}\right)\left(\sum_{s=t-L}^{t-1} a_{t s} \eta_{s}\right)\right\}\right| \\
\leq & 4 q_{n, 1}+4\left(B_{n}^{-2} \sum_{t=1}^{n} \eta_{t}^{2}\left(\sum_{s=1}^{t-L-1} a_{t s} \eta_{s}\right)^{2}\right)^{1 / 2} \\
& \times\left(4 B_{n}^{-2} \sum_{t=1}^{n} \eta_{t}^{2}\left(\sum_{s=t-L}^{t-1} a_{t s} \eta_{s}\right)^{2}\right)^{1 / 2} \leq 4\left(q_{n, 1}+q_{n, 1}^{1 / 2} s_{n, 1}^{1 / 2}\right),
\end{aligned}
$$

where

$$
q_{n, 1}:=B_{n}^{-2} \sum_{t=1}^{n} \eta_{t}^{2}\left(\sum_{s=1}^{t-L-1} a_{t s} \eta_{s}\right)^{2}
$$

Hence, $E\left|s_{n, 2}\right| \leq 4 E q_{n, 1}+4\left(E q_{n, 1} E s_{n, 1}\right)^{1 / 2}$. To bound $E q_{n, 1}$, we argue partly as in (2.10):

$$
E q_{n, 1} \leq C\left\|A_{n}\right\|^{-2} \sum_{t=1}^{n} \sum_{s=1}^{t-L-1} a_{t s}^{2} \rightarrow 0, \quad n \rightarrow \infty, \quad L \rightarrow \infty
$$

by (1.8). We also have

$$
E s_{n, 1} \leq C\left\|A_{n}\right\|^{-2} \sum_{t=1}^{n} \sum_{s=t-L}^{t-1} a_{t s}^{2} \leq C .
$$

Hence $E\left|s_{n, 2}\right| \rightarrow 0$ as $n \rightarrow \infty$ and $L \rightarrow \infty$. This completes the proof of (2.19)(iii) and the theorem.

Acknowledgements Liudas Giraitis and Murad S. Taqqu would like to thank Masanobu Taniguchi for his hospitality in Japan and support by the JSPS grant 15H02061. Murad S. Taqqu was partially supported by the NSF grant DMS-1309009 at Boston University.

Open Access This article is distributed under the terms of the Creative Commons Attribution 4.0 International License (http://creativecommons.org/licenses/by/4.0/), which permits unrestricted use, distribution, and reproduction in any medium, provided you give appropriate credit to the original author(s) and the source, provide a link to the Creative Commons license, and indicate if changes were made.

\section{References}

Bhansali R, Giraitis L, Kokoszka P (2007a) Convergence of quadratic forms with nonvanishing diagonal. Statistics \& Probability Letters 77:726-734

Bhansali R, Giraitis L, Kokoszka P (2007b) Approximations and limit theory for quadratic forms of linear processes. Stochastic Processes and their Applications 117:71-95

Dalla V, Giraitis L, Koul HL (2014) Studentizing weighted sums of linear processes. Journal of Time Series Analysis 35:151-172 
De Jong P (1987) A central limit theorem for generalized quadratic forms. Probability Theory and Related Fields 75:261-277

Giraitis L, Koul HL, Surgailis D (2012) Large Sample Inference for Long Memory Processes. Imperial College Press, London

Guttorp P, Lockhart RA (1988) On the asymptotic distribution of quadratic forms in uniform order statistics. Annals of Statistics 16:433-449

Hall P, Heyde CC (1980) Martingale Limit Theory and Applications. Academic Press, New York

Mikosch T (1991) Functional limit theorems for random quadratic forms. Stochastic Processes and their Applications 37:81-98

Rotar VI (1973) Certain limit theorems for polynomials of degree two. Teoria Verojatnosti i Primenenia 18:527-534 in Russian

Stout W (1974) Almost Sure Convergence. Academic Press, New York 